Yasushi Ido, Yuhiro Iwamoto, Genta Ichinose and Keita Odai

\title{
Transport characteristics of a transport device composed of two magnetically-driven systems using a temperature-sensitive magnetic fluid
}

\begin{abstract}
Transport properties of a fluid transport device composed of two magnetically-driven systems utilizing a temperature-sensitive magnetic fluid were investigated experimentally. The two driving systems were connected in tandem (in series) or in parallel. Their flow rate in the parallel arrangement is larger than in the tandem arrangement. Not only in the case of a single driving system, but also in the case of the two systems connected in either way, the flow rate of the fluid transport device is maximized when the magnet is placed at an appropriate distance from the heating area. When the two systems are connected in tandem, a flow rate of 1.55 times that of the single driving system can be obtained, while in parallel a flow rate of 1.75 times that of the single driving system can be obtained. Using multiple driving systems for fluid transport is therefore a useful method to enhance flow rate.
\end{abstract}

Keywords: ferrofluid, magnetically-driven system, temperature-sensitive magnetic fluid, transport device

Nanotechnology Perceptions 16 (2020) 41-51

doi: 10.4024/N15ID19A.ntp.16.01 